\title{
VMAT-Mediated Changes in Quantal Size and Vesicular Volume
}

\author{
T. L. Colliver, ${ }^{1,2}$ S. J. Pyott, ${ }^{1}$ M. Achalabun, ${ }^{1}$ and Andrew G. Ewing ${ }^{1,2}$ \\ ${ }^{1}$ Department of Chemistry, The Pennsylvania State University, University Park, Pennsylvania 16802, and 2Department of \\ Neuroscience and Anatomy, The Pennsylvania State University College of Medicine, Hershey, Pennsylvania 17033
}

\begin{abstract}
It has been well established that the volume of secretory vesicles can be modulated. However, we present the first data demonstrating that the amount of transmitter in a vesicle can regulate its volume. Amperometry and transmission electron microscopy have been used to determine that L-3,4-dihydroxyphenylalanine and reserpine increase and decrease, respectively, the volume of single pheochromocytoma cell vesicles as well as their catecholamine content. Because changes in vesicular catecholamine content are tracked by changes in vesicle volume, our results
\end{abstract}

indicate that when quantal size is altered via the vesicular monoamine transporter the concentration of catecholamines within the vesicles remains relatively constant. This previously unidentified cellular response provides new insight into how catecholamines can be packaged in and released from secretory vesicles.

Key words: vesicular monoamine transporter; amperometry; transmission electron microscopy; PC12 cells; quantal size; vesicle volume
Amperometry and fast-scan rate cyclic voltammetry (FCV) have proven to be powerful techniques for investigating how the vesicular monoamine transporter (VMAT) can regulate the release of catecholamines from single vesicles at the single cell level (Pothos et al., 1996; Kozminski et al., 1998; Pothos et al., 1998a,b; Travis et al., 2000) Although amperometry can be used to quantitate directly the number of electroactive neurotransmitter molecules released from single vesicles (Wightman et al., 1991; Chow et al., 1992), FCV can be used to determine their concentration (Wightman et al., 1991; Kozminski et al., 1998). Amperometric results at pheochromocytoma (PC12) cells and midbrain dopamine neurons have shown consistently that L-3,4-dihydroxyphenylalanine (LDOPA) increases the quantal amount of released electroactive transmitter(s) (Pothos et al., 1996, 1998a,b). However, a recent study using FCV determined that the relative concentration of electroactive transmitter(s) released from single PC12 cell vesicles is unchanged after exposure to L-DOPA (Kozminski et al., 1998). Additional results using a relatively new method, patch amperometry, also have suggested that the concentration of released neurotransmitter remains constant when quantal size is altered (Albillos et al., 1997).

Assuming the measurements obtained with amperometry and FCV reflect changes in the amount and concentration, respectively, of electroactive transmitter stored within single vesicles before release, then it can be hypothesized that when secretory vesicles increase their neurotransmitter content they also increase their volume. Although vesicle swelling has been shown to occur in a variety of cell types (Finkelstein et al., 1986; Green, 1987), to the best of our knowledge it has yet to be determined whether the amount of neurotransmitter(s) stored within secretory vesicles can affect their volume.

To test this hypothesis, we have used pharmacological manipulations that directly affect the VMAT-mediated transport of catecholamines into PC12 vesicles. Transmission electron microscopy (TEM) has been used to measure changes in the size of PC12 vesicles, and these measurements have been correlated with the

\section{Received April 7, 2000; accepted May 5, 2000}

This work was supported by National Institutes of Health. T.L.C. is supported in part by a National Institute of Mental Health predoctoral fellowship. We thank Drs. David Sulzer, Emmanuel Pothos, and Robert Chow for their helpful discussions and suggestions; Ami Frank for her cell culture expertise; and Rosemary Walsh for her assistance with the TEM images.

Correspondence should be addressed to Dr. Andrew G. Ewing, Department of Chemistry, 152 Davey Laboratory, The Pennsylvania State University, University Park, PA 16802-6300. E-mail: age@psu.edu.

Copyright $\odot 2000$ Society for Neuroscience $0270-6474 / 00 / 205276-07 \$ 15.00 / 0$ amount of neurotransmitter released by using amperometry. In this study we demonstrate that L-DOPA and reserpine can increase and decrease, respectively, quantal size and the volume of dense core vesicles in PC12 cells. Almost all of the volume changes under these conditions can be attributed to changes in the volume of the electron-lucent halo surrounding the dense core. These results indicate that the amount released from individual vesicles is related directly to their volume before secretion and suggest that vesicles regulate a constant concentration of neurotransmitter. It is proposed that water moving in and out of PC12 vesicles with changing amounts of catecholamines causes changes in vesicle volume and maintains catecholamines at a constant concentration. The physiological consequences of these findings in synaptic release are discussed also.

\section{MATERIALS AND METHODS}

Cell culture. Stock PC12 cells were generously provided by Dr. Dave Sulzer (Columbia University, New York, NY) and maintained as described previously (Kozminski et al., 1998). In brief, PC12 cells were grown on mouse collagen IV-coated culture dishes (Becton Dickinson, Bedford, MA) in RPMI-1640 medium supplemented with horse serum, fetal bovine serum, and penicillin/streptomycin. Cells were kept in a $7 \% \mathrm{CO}_{2}$ atmosphere at $37^{\circ} \mathrm{C}$ and were subcultured approximately every $7-9 \mathrm{~d}$ or when confluency was reached. Cells were used for all experiments between days 7 and 12 of subculturing.

Electrode preparation and experimental setup. Carbon fiber microelectrodes $(5 \mu \mathrm{m}$ diameter) were constructed as described previously (Pothos et al., 1998b) and back-filled with $3 \mathrm{M} \mathrm{KCl}$. Electrode tips were polished at a $45^{\circ}$ angle on a diamond dust-embedded micropipette beveling wheel (Model BV-10, Sutter Instrument, Novato, CA). Cyclic voltammograms were generated for each electrode in a nitrogen-saturated $0.1 \mathrm{~mm}$ dopamine solution (in $0.1 \mathrm{M}$ PBS, pH 7.4), and only electrodes with stable $I-E$ curves were used.

Cells were prepared for an experiment by removing the culture medium and adding $15 \mathrm{ml}$ of physiological saline [containing (in $\mathrm{mm}$ ) $150 \mathrm{NaCl}, 5$ $\mathrm{KCl}, 1.2 \mathrm{MgCl}_{2}, 5$ glucose, 10 HEPES, and $2 \mathrm{CaCl}_{2}, \mathrm{pH}$ 7.4] and then placing them onto the stage of an inverted microscope (IM-35, Carl Zeiss, Thornwood, NY). The working electrode was lowered gently onto a single cell by using a piezomicropositioner (PCS-750/1000, Burleigh Instruments, Fishers, NY). The close proximity of the electrode to the cell surface was confirmed by a slight deformation in the outline of the cell.

Exocytosis was stimulated at $\sim 30 \mathrm{sec}$ intervals with a $5 \mathrm{sec}, 20 \mathrm{psi}$ pulse (Picospritzer II, General Valve, Fairfield, NJ) of physiological saline with $100 \mathrm{mM} \mathrm{K}^{+}$from a micropipette cut to $\sim 10 \mu \mathrm{m}$ and positioned $55-70 \mu \mathrm{m}$ from a cell. The concentration of $\mathrm{NaCl}$ in the elevated $\mathrm{K}^{+}$solution was adjusted to maintain isotonicity. All cells were inspected visually after recording to verify that the cell had not been damaged. All experiments were performed at $37 \pm 1^{\circ} \mathrm{C}$. Culture dishes were warmed with a solid-state Peltier heating device (Bionomic System, 20/20 Technology, Wilmington, NC).

Data acquisition and data analysis. Electrodes were held at $+0.65 \mathrm{~V}$ versus a locally constructed sodium-saturated calomel reference electrode that used a commercially available patch-clamp instrument (Axopatch 
200B; Axon Instruments, Foster City, CA) configured as described previously (Borges et al., 1997). The output was digitized at $5 \mathrm{kHz}$ and filtered at $2 \mathrm{kHz}$ by using an internal four-pole low-pass Bessel filter. Data were displayed in real time (Axoscope 1.1.1.14, Axon Instruments) and stored to a computer with no subsequent filtering.

Exocytotic spikes were identified, and the spike characteristics-area (pC), $t_{1 / 2}(\mathrm{msec})$, and $I_{\max }(\mathrm{pA})$ - were determined by using a multi-pass algorithm described previously (Schroeder et al., 1992). Signals were designated as spikes if their $I_{\text {max }}$ values were six times the SD (typically 0.7 $\mathrm{pA}$ ) of a $1 \mathrm{sec}$ portion of stable baseline recorded before the first stimulation. All peaks identified by the program were inspected visually, and overlapping peaks were excluded manually from the data sets. Additionally, if a spike had a zero correlation with an exponentially modified gaussian function, it also was excluded (Jankowski et al., 1994; Schroeder et al., 1996). On average, this was $\sim 5 \%$ of the well resolved spikes.

Amperometry experiments. A same-cell paradigm for the amperometry experiments has been applied. The same electrode was used to measure release from a cell before and after drug treatment. This approach minimizes cell-to-cell and electrode variability and, as a result, should be more sensitive to changes in spike characteristics as compared with measuring release from separate groups of cells (Pothos et al., 1998a; Colliver et al., 2000). Additionally, with this technique it is possible to record from a cell within a fairly consistent time after treatment.

Data for the spike characteristics of area, $t_{1 / 2}$, and $I_{\max }$ are reported as ratio values created from the same $\mathrm{PC} 12$ cell before and after it was treated with drug. Each cell was stimulated at least twice, and the position of the electrode and injector relative to the cell were recorded. Then both were lifted above the cell and out of solution, and the cells were exposed to 100 $\mu \mathrm{M}$ L-DOPA, $100 \mathrm{nM}$ reserpine, or both by adjusting the physiological saline surrounding the cells. Drugs were dissolved in either physiological saline or dimethylsulfoxide (reserpine). After a 90 min incubation period the cells were rinsed with physiological saline without drug and were allowed to rewarm to $37^{\circ} \mathrm{C}$. Postdrug measurements usually were obtained 15-17 min after rinsing by repositioning the working electrode and injector to their original location.

Histograms of raw spike characteristics are skewed heavily to the right. Because means from normal distributions should be less biased by outliers, we have created mean spike ratios from log-transformed values (Pothos et al., 1998b; Colliver et al., 2000). To create spike characteristic ratios, we determined the area, $t_{1 / 2}$, and $I_{\max }$ values at a cell before (pre) and after (post) the incubation period. Then ratios for each characteristic were created by dividing the mean of log values after the incubation period by the mean of $\log$ values before treatment (i.e., mean of $\log$ post $/$ mean of $\log$ pre). To obtain a reasonable estimate of pre and post mean spike values, we rejected any experiment in which at least 15 well resolved events were not measured from a cell after the first two stimulations that followed drug treatment. Additionally, to ensure reliable placement of the electrode, we used only cells that maintained their morphology during the incubation period.

Transmission electron microscopy (TEM). PC12 cells were treated with $100 \mu \mathrm{M}$ L-DOPA, $100 \mathrm{~nm}$ reserpine, or both of these drugs in physiological saline or with only physiological saline (controls) for $90 \mathrm{~min}$ at $37^{\circ} \mathrm{C}$. Then the cells were rinsed with PBS, pH 7.4, and treated with $0.05 \%$ trypsinEDTA (Life Technologies, Gaithersburg, MD) for $30 \mathrm{sec}$. The trypsinEDTA solution was replaced with PBS, and the cells were dispersed into solution by flushing them off the culturing substrate. Single cell suspensions were transferred to Microfuge tubes and pelleted at $100 \times g$ for $10 \mathrm{~min}$. Cell pellets were prepared for transmission electron microscopy (TEM) by using conventional chemical preparation (Dykstra, 1992). Specifically, pellets were incubated overnight at $4^{\circ} \mathrm{C}$ in Karnovsky's fixative (Karnovsky, $1965)$, osmicated $\left(1 \%\right.$ aqueous $\left.\mathrm{OsO}_{4}\right)$, stained with uranyl acetate $(2 \%$ aqueous), dehydrated in a graded series of ethanol and acetone, and embedded in Spurr's resin at $60^{\circ} \mathrm{C}$ for $48 \mathrm{hr}$. Thin sections $(60 \mathrm{~nm})$ were obtained on a Leica ultramicrotome (LKB III-8800, Ultracut E, Deerfield, IL) with a diamond knife (Diatome, Fort Washington, PA). Sections were counterstained with uranyl acetate and lead citrate (Venable and Coggeshall, 1965) before being viewed on a transmission electron microscope (1200EXII, JEOL, Peabody, MA) at $80 \mathrm{kV}$.

Quantitative analysis of vesicle structures was performed with SigmaScan (Version 5.0.0, SPSS, Chicago, IL). TEM images were imported into this software, and the limiting membrane of each vesicle and the perimeter of its dense core were traced. Once each object was inscribed, SigmaScan determined its diameter and cross-sectional area. Diameter was defined as the distance between the two most distal points on the initial trace. Only those cells in which at least part of the cell membrane and nucleus could be observed were considered to be "intact" and suitable for morphometric analysis. Additionally, only cells in which a minimum of 15 vesicles could be counted were used. In PC12 cells the vesicular monoamine transporter is preferentially localized to dense core vesicles (Liu et al., 1994). Because electroactive monoamines are the only type of neurotransmitter(s) that can be detected from PC12 cells by using amperometry, only vesicles in which a dense core could be identified clearly were measured.

Initial experiments were performed to investigate the effects of L-DOPA on the volume of PC12 vesicles. For these experiments only sister cultures either with or without L-DOPA were prepared for imaging analysis. For subsequent experiments vesicle volume measurements also were acquired from cells exposed only to reserpine or to both reserpine and L-DOPA. To

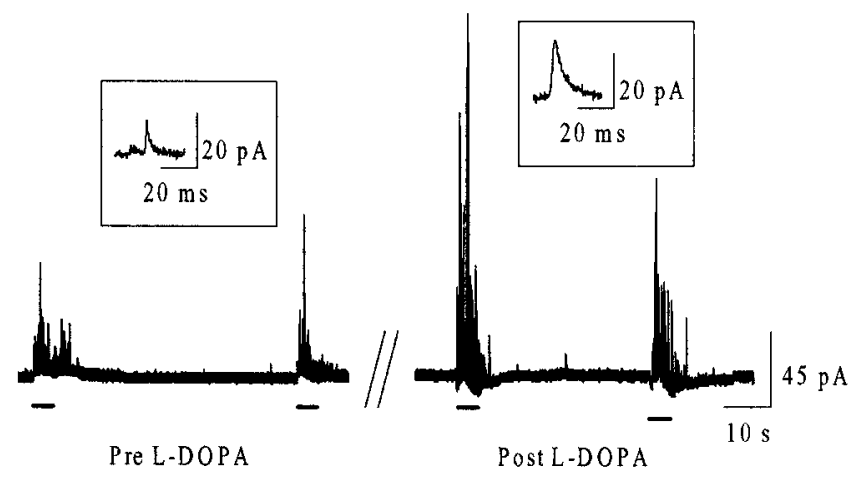

Figure 1. Representative amperometric data from a single PC12 cell before and after exposure to $100 \mu \mathrm{M} \mathrm{L}$-DOPA for $90 \mathrm{~min}$. Insets above each of the main traces display individual events at smaller time and current scales. These individual spikes approximate the mean area, $t_{1 / 2}$, and $I_{\max }$ values measured before and after treatment with L-DOPA. Bars under each of the main traces represent the time and duration of stimulus $\left(100 \mathrm{~mm} \mathrm{~K}^{+}\right)$ application.

control for variabilities in the fixation method and the different ages of the cells that were used (Doupe et al., 1985), we processed separate controls in parallel for cells under these treatment conditions.

Volume values were calculated on the basis of the simplified assumption that the vesicle structures were spherical. This assumption had no impact on the results because the same statistical trends were observed when either diameters or cross-sectional areas were compared between groups of cells (data not shown). Additionally, because vesicle measurements in each treatment group were compared with separate controls, no corrections for factors such as plane of section were made (Coupland, 1968). As a result, the measured changes in volume reflect relative changes in vesicle morphology.

Statistical analysis. To ensure that cells with a large number of vesicles would not be over-represented within a treatment group, we calculated mean values for vesicle structures from each cell, and we statistically compared samples of mean values for different treatment groups, instead of pooled samples (Colliver et al., 2000). Amperometric spike ratios and means of vesicle structures were tested for significant differences by using the Student's $t$ test (SigmaStat Version 2.03, SPSS). Results for all tests were considered significant if associated $p$ values were $<0.05$. All values are reported as the mean \pm SEM; all plots were created with SigmaPlot (Version 4.00, SPSS).

\section{RESULTS}

\section{VMAT-mediated changes in quantal release}

We have used a same-cell paradigm for amperometry experiments in which the same electrode is used to measure release from a cell before and after drug exposure. Ratios for spike area, $t_{1 / 2}$, and $I_{\max }$ were created from a cell by dividing the mean of log values after the incubation period by the mean of log values before treatment (see Materials and Methods). Peak area is defined as the time integral of each current transient, $I_{\max }$ is the height of each peak, and $t_{1 / 2}$ is the width of each peak at one-half of its height. The area of each amperometric spike is directly proportional to the number of molecules detected from a single vesicle by the relationship $Q=$ $n N F$, where $Q$ is the charge of each current transient, $N$ is the number of moles, $F$ is Faraday's constant (96,485 C/equiv), and $n$ is the number of electrons transferred per oxidized molecule (this was assumed to be 2 for catecholamines). The values $I_{\max }$ and $t_{1 / 2}$ have been shown to be dependent on several factors, including the number of molecules released (Pothos et al., 1996, 1998a), the degranulation and extrusion of transmitter from the vesicle lumen (Wightman et al., 1995; Pihel et al., 1996; Borges et al., 1997), and the type and magnitude of filtering used during the recording.

Example data from a PC12 cell before (pre) and after (post) exposure to L-DOPA for $90 \mathrm{~min}$ are shown in Figure 1. In agreement with previous work, exposure of PC12 cells to $100 \mu \mathrm{M}$ L-DOPA for 90 min significantly increased spike area and $t_{1 / 2}$ values (Fig. 2) (Pothos et al., 1996). Although $I_{\max }$ ratios appeared to be increased by L-DOPA, this change was not significant when compared with that measured at control cells. When PC12 cells were treated for $90 \mathrm{~min}$ with $100 \mathrm{~nm}$ reserpine, a potent inhibitor of the 
Control L-DOPA Reserpine L-DOPA \& Reserpine
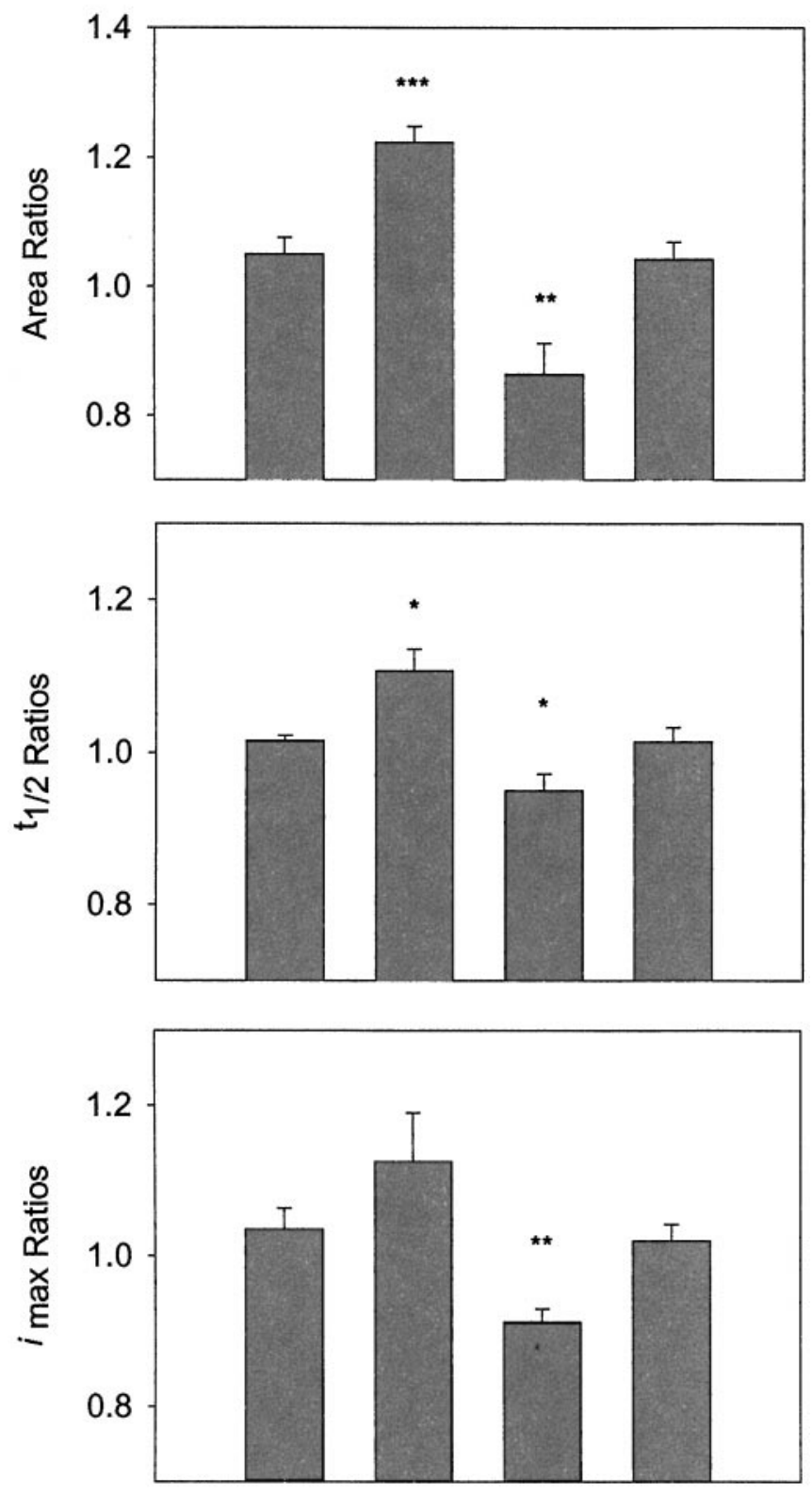

Figure 2. Summary of spike characteristic ratio values created at individual PC12 cells. Ratios for each characteristic were created at a cell by dividing the mean of log values after the incubation period by the mean of log values before treatment (see Materials and Methods). Ratios created from control cells (i.e., cells not exposed to drug during the $90 \mathrm{~min}$ incubation period) yielded ratio values close to 1 . Under control conditions there was an average of $\sim 194,000 \pm 13$ molecules released per vesicle. This value is slightly greater than those reported previously [114,300 (Chen et al., 1994); 74,736 (Pothos et al., 1996)] and could be attributable to differences in culturing protocols and/or experimental conditions. For the ratios presented, an average of $159 \pm 29$ and $104 \pm 13$ amperometric values was used to determine pre and post means, respectively. Error bars represent the mean \pm SEM of spike characteristic ratio values for the different experimental conditions (Control, $n=6 ; L-D O P A, n=7$; Reserpine, $n=5$; $L-D O P A \&$ Reserpine, $n=5$ ). Values marked with $* * *$ are statistically different with $p<0.001$, **significant difference with $p<0.01$, and *significant difference with $p<0.05$ versus control (Student's $t$ test).

VMAT (Varoqui and Erickson, 1997), there was a significant decrease in peak area, $t_{1 / 2}$, and $I_{\max }$ values (Fig. 2). Dimethylsulfoxide, the vehicle used for reserpine administration, had no significant effect on any of the spike characteristics $(n=3$; data not shown). In contrast to the effects independently elicited by both drugs, when PC12 cells were exposed simultaneously to $100 \mu \mathrm{M}$ L-DOPA and $100 \mathrm{~nm}$ reserpine for $90 \mathrm{~min}$, spike characteristic ratios were not significantly different from those measured at control cells (Fig. 2).

\section{VMAT-mediated changes in vesicular volume}

Representative TEM images from single PC12 cells are shown in Figure 3. For all of the cells that were examined, dense core vesicles could be observed readily throughout the cytoplasm. Additionally, for all of the vesicles that were analyzed, a limiting membrane could be discerned clearly from the dense core. In general, the dense core vesicles resembled those described for PC12 cells prepared in a similar manner (Watanabe et al., 1983; Pozzan et al., 1984; Madeddu et al., 1985; Schafer et al., 1987; Hase et al., 1996).

The mean diameter of the outer limiting membrane for all control dense core vesicles was $198 \pm 9 \mathrm{~nm}$. This value is similar to those reported previously for PC12 cells (Schubert and Klier, 1977; Schubert et al., 1980). Overall, the volume of PC12 dense core vesicles tracked the changes in quantal size observed in response to L-DOPA and reserpine (compare Figs. 2, 4). When PC12 cells were treated with $100 \mu \mathrm{m} \mathrm{L-DOPA}$ for $90 \mathrm{~min}$, there was a dramatic increase in the volume of dense core vesicles (see Fig. 3b). This increase was significant when compared with that of control values (Fig. 4) and corresponded to a $34 \%$ increase in vesicle diameter or a $141 \%$ increase in volume (relative to separate controls). Similar changes were observed when cells were treated with $100 \mu \mathrm{M}$ L-DOPA for $30 \mathrm{~min}$, although the change was of a slightly smaller magnitude (data not shown). In contrast to the effects of L-DOPA, PC12 dense core vesicles were observed to decrease significantly in size after treatment with $100 \mathrm{~nm}$ reserpine for $90 \mathrm{~min}$ (see Figs. 3c, 4.). Similar to the results found with amperometry, there was no significant change in vesicle volume when PC12 cells were treated simultaneously with $100 \mu \mathrm{M}$ L-DOPA and $100 \mathrm{nM}$ reserpine for 90 min (see Figs. 3d, 4).

For each dense core vesicle that was measured, a space between the dense core and the limiting membrane could be identified. For simplicity, this structural component of the dense core vesicles will be referred to as the vesicle halo. To investigate changes independently in the volume of the dense core and vesicle halo for the data presented above, we calculated halo volumes by subtracting the volume of the dense core from that of the entire vesicle. For clarity, dense core and halo volume measurements for the different treatment groups have been plotted separately with their respective controls in Figure 5. The decreased volumes observed for the second control group in Figure 5 are consistent with the fact that these cells were prepared for analysis at a later point in time (Doupe et al., 1985). Although L-DOPA and reserpine were found to increase and decrease the volume of the vesicle halo, respectively, neither drug significantly affected the volume of the dense core (Fig. 5). There was no significant change in either the vesicle halo or dense core volumes when PC12 cells were exposed simultaneously to L-DOPA and reserpine (Fig. 5).

\section{Estimating vesicular catecholamine concentration}

When the average number of moles of neurotransmitter detected from control PC12 vesicles is combined with the average volume of all control vesicles, a concentration of $80 \mathrm{~mm}$ is obtained. Mean amperometric and TEM values combined from cells exposed for 90 min to L-DOPA, reserpine, and L-DOPA and reserpine yield concentration values of 65,93 , and $118 \mathrm{~mm}$, respectively. These values are all close to $99 \mathrm{~mm}$, previously estimated for untreated PC12 cells (Finnegan et al., 1996).

Although all of the estimated concentration values are similar in magnitude, it is not possible to compare single point estimates statistically for each experimental condition. Therefore, to investigate possible changes in concentration, we have plotted the mean number of neurotransmitter moles detected against the mean volume of PC12 vesicles. As shown in Figure 6, there is a direct relationship between the mean vesicle volume and the mean amount of neurotransmitter released. Assuming that changes in 

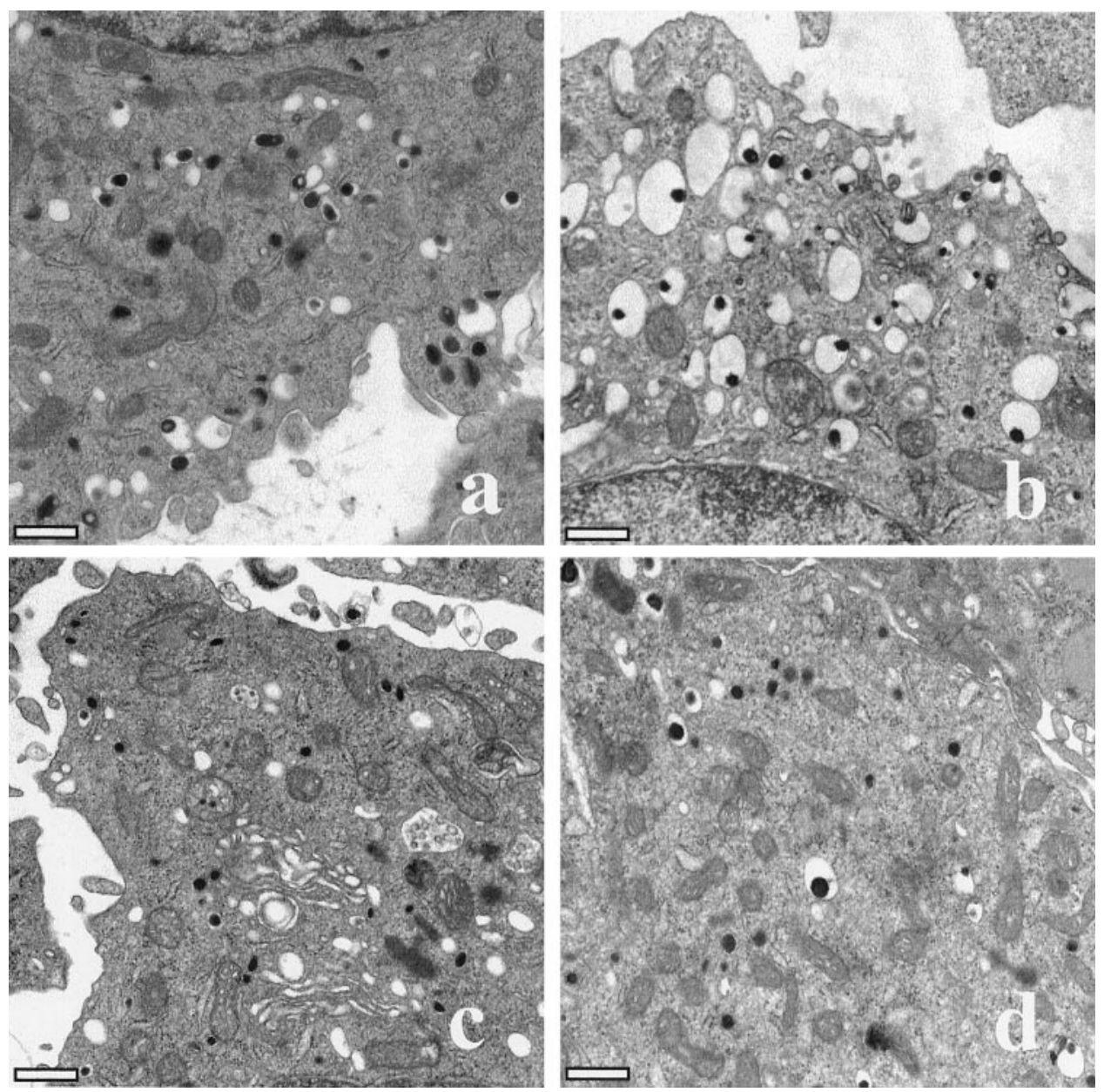

Figure 3. Representative TEM images from single PC12 cells after treatment with $(a)$ physiological saline, $(b) 100 \mu \mathrm{M} \mathrm{L}-\mathrm{DOPA},(c)$ $100 \mathrm{~nm}$ reserpine, and $(d) 100 \mu \mathrm{M}$ L-DOPA and $100 \mathrm{~nm}$ reserpine for $90 \mathrm{~min}$. A portion of the nucleus can be seen for the cells shown in $a$ and $b$ (at the top and bottom, respectively). Scale bars, $500 \mathrm{~nm}$. quantal size reflect changes in the amount of neurotransmitter(s) stored within PC12 cell vesicles, these results suggest that PC12 vesicles can adjust their volume when accommodating different amounts of neurotransmitter to regulate their neurotransmitter concentration.

\section{DISCUSSION}

\section{Effects of L-DOPA and reserpine on quantal size and vesicular volume}

Treatment of PC12 cells with L-DOPA increases the volume of dense core vesicles as measured by TEM (see Figs. 3b, 4). If L-DOPA stimulates exocytosis, then it is possible that the increase in vesicle volume that is observed after treatment with L-DOPA could result from the influx of extracellular solution into the vesicles during membrane fusion (Breckenridge and Almers, 1987; Zimmerberg et al., 1987). However, on the basis of the morphological criteria used in previous studies of PC12 cells, all vesicle measurements were felt to reflect the morphology of dense core vesicles that were not actively involved in exocytosis (Watanabe et al., 1983; Madeddu et al., 1985; Hase et al., 1996). Most notable were the extremely rare vesicle-plasmalemmal appositions and the complete lack of exocytotic profiles. These findings are not unexpected, because the fusion process is very rapid and presumably difficult to catch (Watanabe et al., 1983; Madeddu et al., 1985). Our results, therefore, are inconsistent with a mechanism in which vesicles swell as a result of undergoing exocytosis, and so another mechanism has been considered.

As shown in Figures 2 and 4, reserpine blocks the increase in quantal size and vesicle volume caused by L-DOPA. These results confirm that the changes in quantal size and vesicle volume are VMAT-mediated. Because L-DOPA can augment intracellular DA levels (Pothos et al., 1996), the observed increases in quantal size

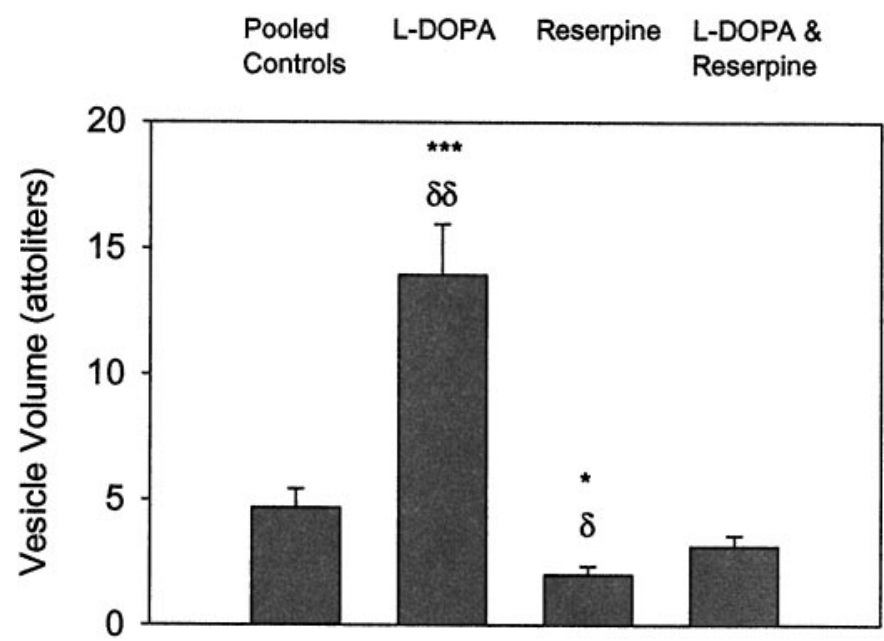

Figure 4. Summary of mean volume changes in PC12 dense core vesicles treated with L-DOPA and/or reserpine. Although vesicle volumes were determined for a total of five separate groups of cells (i.e., two control groups; see Materials and Methods), measurements from the separate control groups have been combined and labeled Pooled Controls in this figure so that the changes in vesicle volume can be compared directly with the amperometry results shown in Figure 2. Error bars represent mean \pm SEM of mean volume values from all control cells $(n=23)$, cells exposed to L-DOPA $(n=10)$ or reserpine $(n=9)$, or both simultaneously $(n=9)$. An average of $42 \pm 3$ vesicles was measured per cell. Values marked with *** are statistically different with $p<0.001$; *indicates a significant difference with $p<0.05$ versus pooled controls (Student's $t$ test). Those values marked with $\delta \delta$ and $\delta$ are significantly different from separate controls, with $p<0.01$ and $p<0.05$, respectively. Although mean values are shown in this figure, the same statistical trends were observed when the data were pooled from each group and compared statistically. 


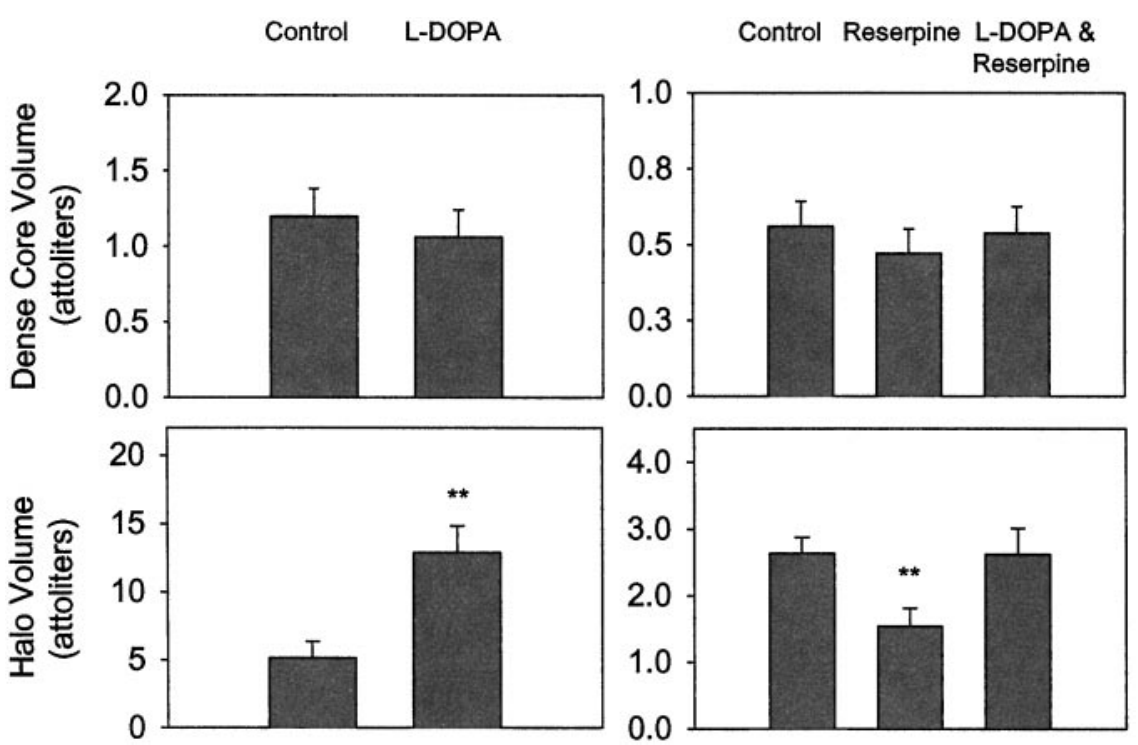

Figure 5. Summary of the effects of L-DOPA and/or reserpine on mean vesicle halo and dense core volume. The control values for L-DOPA-treated cells were measured from a total of 11 cells, and the second control group (i.e., for reserpine) consists of values from 12 different cells. The number of cells used for the other groups and the average number of vesicles measured per cell to calculate dense core and halo volumes are given in the legend to Figure 4. Error bars represent the mean \pm SEM of mean vesicle halo and dense core volumes. Values marked with $* *$ are statistically different, with $p<0.01$ versus controls (Student's $t$ test). Although mean values are shown in this figure, the same statistical trends were observed when the data were pooled from each group and statistically compared.

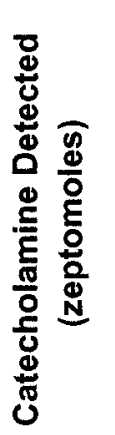

1000
800
600
400
200
0

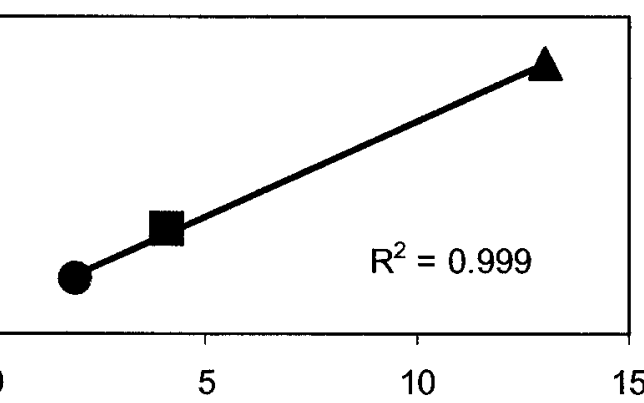

Vesicle Volume (attoliters)

Figure 6. Correlation between the amount of neurotransmitter detected and the volume of PC12 dense core vesicles. Point estimates for moles of catecholamine released per vesicle and vesicle volume were determined from PC12 cells after treatment with $100 \mu \mathrm{M} \mathrm{L}-\mathrm{DOPA}(\boldsymbol{\Delta}), 100 \mathrm{~nm}$ reserpine (O), or only physiological saline (ם) for 90 min. After treatment with reserpine, one cell continued to release an unusually large amount of neurotransmitter with an apparent concentration of $450 \mathrm{~mm}$ per vesicle. This cell was excluded for these comparisons. Amount and volume values measured from PC12 cells simultaneously exposed to L-DOPA and reserpine are similar in magnitude to those measured from control cells. For clarity, these values have been excluded from this figure. When amount and volume values measured from PC12 cells simultaneously exposed to L-DOPA and reserpine for $90 \mathrm{~min}$ and to only L-DOPA for $30 \mathrm{~min}$ are combined with those shown above (i.e., for a total of five treatment conditions), the calculated correlation coefficient is 0.892 .

and vesicle volume are likely attributable to the uptake of excess cytoplasmic neurotransmitter via the VMAT. When used alone, reserpine decreases vesicle volume and quantal size.

Reserpine is known to block catecholamine uptake by binding to the VMAT (Varoqui and Erickson, 1997). Recently, two types of neurotransmitter efflux from isolated rat synaptic vesicles have been described: one that is dependent on neurotransmitter uptake by the VMAT and one that is not (Floor et al., 1995). The decrease in quantal size and vesicle volume in the presence of reserpine presumably results from the persistence of an uptake-independent efflux of neurotransmitter. Although reserpine blocked the L-DOPA-mediated increase in quantal size and vesicular volume, L-DOPA also blocked the extent to which reserpine could decrease the transmitter content and volume of PC12 vesicles (see Figs. 2, 4). L-DOPA increases intracellular levels of monoamines in PC12 cells (Pothos et al., 1996). The decreased ability of reserpine to deplete vesicles in the presence of L-DOPA may be attributable to the fact that binding of reserpine to the VMAT can be inhibited competitively by concentrations of monoamines in the low micromolar

range (Scherman and Henry, 1984; Varoqui and Erickson, 1997). When cells are exposed to reserpine alone, monoamine levels are presumably lower and less capable of competing with reserpine for the VMAT.

\section{Mechanisms regulating vesicle volume and catecholamine concentration}

Dense core vesicles of adrenal chromaffin cells contain high (mM) concentrations of catecholamines, ATP, and ions such as $\mathrm{Ca}^{2+}$ (Winkler and Westhead, 1980). In adrenal chromaffin vesicles these high concentrations are partially maintained by associations between small molecules and ions and the soluble acidic proteins within the vesicle matrix (Kopell and Westhead, 1982; Yoo and Albanesi, 1991; Videen et al., 1992). By reducing the number of free molecules and ions within solution, these associations provide a mechanism for reducing the intravesicular osmotic pressure, facilitating the storage of high concentrations of transmitter within the small volume of the vesicle (Kopell and Westhead, 1982; Haigh et al., 1989). Because PC12 cells are derived from the rat adrenal gland (Greene and Tischler, 1976) and the structure and composition of dense core vesicles in these cells resemble those found in normal chromaffin cells (Tischler and Greene, 1978; Wagner, 1985; Fischer-Colbrie and Schober, 1987), it is reasonable to assume they store catecholamines in much the same way.

As shown in Figures 2 and 5, the volume of the halo of dense core vesicles is affected directly by changing amounts of catecholamines within PC12 vesicles. If binding sites within the dense core are saturated normally, then, as suggested by Figure 5, excess transmitter accumulated by $\mathrm{PC} 12$ cell vesicles would be stored outside of the dense core. Experiments with dense core vesicles from chromaffin, mast, and splenic nerve cells in hypotonic solutions have demonstrated that vesicles can accumulate water and increase their volume to keep their contents in osmotic equilibrium with their environment (Thureson-Klein et al., 1975; Sudhof, 1982; Brodwick et al., 1992). On the basis of these findings, it is possible that after exposure to L-DOPA the increased levels of osmotically active material within PC12 vesicles lead to the uptake of water and an increase in vesicle volume. Similar mechanisms could be used to explain the decrease in vesicle volume caused by reserpine.

Balancing the amount of water within $\mathrm{PC} 12$ vesicles with the amount of excess catecholamine would keep the vesicle interior in osmotic equilibrium with the surrounding cytoplasm. More importantly, proportional changes in the water content of the vesicles with changing levels of catecholamines would ensure that the vesicular catecholamine concentration remains constant when catecholamine levels are altered (Fig. 6). A physiological advantage to keeping the vesicular catecholamine concentration constant during 
vesicle loading is that the VMAT does not have to work against a continually increasing concentration gradient.

Using large lecithin vesicles, Kwok and Evans have shown that the bilayer membrane of a vesicle can rupture once its surface area increases by $\sim 2-3 \%$ (i.e., a $1.5 \%$ change in diameter; Kwok and Evans, 1981). However, results presented here and in previous studies (Zimmerberg et al., 1987; Brodwick et al., 1992; Jena et al., 1997) clearly demonstrate that vesicles can increase their volume beyond this limit. In one account for the apparent discrepancies between observed and predicted limits for increases in vesicle volume, it has been suggested that the membrane of dense core vesicles is flaccid against the dense core before swelling and that the extra membrane is used during membrane expansion (Zimmerberg et al., 1987; Brodwick et al., 1992).

Alternatively, EM studies of adrenal cell vesicles have identified small lipid vesicles present between the dense core and the vesicle membrane (Ornberg et al., 1986); it has been hypothesized that these structures contribute material to vesicle membranes when they expand (Ornberg et al., 1995). Regardless of the mechanism(s) used by PC12 vesicles to increase their volume, it appears that there is a limit to which vesicles can expand (Kwok and Evans, 1981; VanderMeulen and Grinstein, 1982). Therefore, it is possible that the physical properties of vesicle membranes also may be an important factor in the modulation of quantal size.

\section{Implications in synaptic release}

Understanding communication between mammalian neurons is the ultimate goal of most neuronal studies. Although postsynaptic recordings have demonstrated that changes in quantal release affect postsynaptic responses (Van der Kloot, 1987; Song et al., 1997), the relatively large size of electrodes currently available makes it difficult to determine how changes in quantal release affect the synaptic environment. However, results with FCV at PC12 cells have shown that, after exposure to L-DOPA, the relative concentration of vesicular transmitter released is unchanged when compared with that of untreated cells (Kozminski et al., 1998). If the concentration of transmitter released from individual vesicles remains constant in the synapse when quantal size is altered, then it is unclear how changes in quantal size alter postsynaptic responses.

Using the dimensions for a typical dopamine synapse found in the rat neostriatum, we can show that the distance across the synaptic gap is similar to the diameter of the synaptic vesicle (Pickel et al., 1981). If it is assumed that the contents of a vesicle are released into the synapse faster than they can be diluted, then because of the restricted volume within the synapse, material released from a vesicle would experience little initial dilution and would move rapidly away from the point of release at a relatively constant concentration. Using an estimate of 7000 molecules per quantal event (Pothos et al., 1998a) and an average vesicle radius of $25 \mathrm{~nm}$ (Pickel et al., 1981), the concentration of dopamine within and released from single dopamine vesicles would be $\sim 75 \mathrm{~mm}$. Because this concentration is much higher than the $K_{\mathrm{D}}$ for dopamine receptors (low nM; O'dowd et al., 1994), it appears that even under normal conditions synaptic dopamine receptors are saturated after a release event.

If synaptic vesicles completely release their contents during exocytosis, then an increase in quantal size would increase the vesicular volume and the volume of concentrated neurotransmitter released into the synapse immediately after an exocytotic event. Because of the larger volume of concentrated neurotransmitter in the synapse, an increase in quantal size would increase the number of postsynaptic receptors initially activated. Using similar reasoning, a decrease in quantal size may result in fewer receptors initially activated after a release event. Therefore, the model presented here is that the volume of transmitter released in each exocytotic event, and not the concentration, dictates the number of postsynaptic receptors initially activated. This suggests that the concentration of released neurotransmitter is not important until after the initial response and perhaps not until the transmitter escapes the synapse on its way to affecting other nearby cells (Garris et al., 1994).

\section{REFERENCES}

Albillos A, Dernick G, Horstmann H, Almers W, Alvarez de Toledo G, Lindau M (1997) The exocytotic event in chromaffin cells revealed by patch amperometry. Nature 389:509-512.

Borges R, Travis ER, Hochstetler SE, Wightman RM (1997) Effects of external osmotic pressure on vesicular secretion from bovine adrenal medullary cells. J Biol Chem 272:8325-8331.

Breckenridge LJ, Almers W (1987) Final steps in exocytosis observed in a cell with giant secretory granules. Proc Natl Acad Sci USA 84:1945-1949.

Brodwick MS, Curran M, Edwards C (1992) Effects of osmotic stress on mast cell vesicles of the beige mouse. J Membr Biol 126:159-169.

Chen TK, Luo G, Ewing AG (1994) Amperometric monitoring of stimulated catecholamine release from rat pheochromocytoma (PC12) cells at the zeptomole level. Anal Chem 66:3031-3035.

Chow RH, von Ruden L, Neher E (1992) Delay in vesicle fusion revealed by electrochemical monitoring of single secretory events in adrenal chromaffin cells. Nature 356:60-63.

Colliver TL, Hess EJ, Pothos EN, Sulzer D, Ewing AG (2000) Quantitative and statistical analysis of the shape of amperometric spikes recorded from two populations of cells. J Neurochem 74:1086-1097.

Coupland RE (1968) Determining sizes and distribution of sizes of spherical bodies such as chromaffin granules in tissue sections. Nature 217:384-388.

Doupe AJ, Landis SC, Patterson PH (1985) Environmental influences in the development of neural crest derivatives: glucocorticoids, growth factors, and chromaffin cell plasticity. J Neurosci 5:2119-2142.

Dykstra MJ (1992) Biological electron microscopy: theory, techniques, and troubleshooting. New York: Plenum.

Finkelstein A, Zimmerberg J, Cohen FS (1986) Osmotic swelling of vesicles: its role in the fusion of vesicles with planar phospholipid bilayer membranes and its possible role in exocytosis. Annu Rev Physiol 48:163-174.

Finnegan JM, Pihel K, Cahill PS, Huang L, Zerby SE, Ewing AG, Kennedy RT, Wightman RM (1996) Vesicular quantal size measured by amperometry at chromaffin, mast, pheochromocytoma, and pancreatic $\beta$-cells. J Neurochem 66:1914-1923.

Fischer-Colbrie R, Schober M (1987) Isolation and characterization of chromogranins $\mathrm{A}, \mathrm{B}$, and $\mathrm{C}$ from bovine chromaffin granules and a rat pheochromocytoma. J Neurochem 48:262-270.

Floor E, Leventhal PS, Wang Y, Meng L, Chen W (1995) Dynamic storage of dopamine in rat brain synaptic vesicles in vitro. J Neurochem 64:689-699.

Garris PA, Ciolkowski EL, Pastore P, Wightman RM (1994) Efflux of dopamine from the synaptic cleft in the nucleus accumbens of the rat brain. J Neurosci 14:6084-6093.

Green DP (1987) Granule swelling and membrane fusion in exocytosis. J Cell Sci 88:547-549.

Greene LA, Tischler AS (1976) Establishment of a noradrenergic clonal line of rat adrenal pheochromocytoma cells which respond to nerve growth factor. Proc Natl Acad Sci USA 73:2424-2428.

Haigh JR, Parris R, Phillips JH (1989) Free concentrations of sodium, potassium and calcium in chromaffin granules. Biochem J 259:485-491.

Hase T, Jett M, Asafo-Adjei E, Topper M (1996) Release of chromaffin granular content from staphylococcal enterotoxin B (SEB)-treated and -untreated PC12 cells. In Vitro Cell Dev Biol Anim 32:322-328.

Jankowski JA, Finnegan JM, Wightman RM (1994) Extracellular ionic composition alters kinetics of vesicular release of catecholamines and quantal size during exocytosis at adrenal medullary cells. J Neurochem 63:1739-1747.

Jena BP, Schneider SW, Geibel JP, Webster P, Oberleithner H, Sritharan $\mathrm{KC}$ (1997) $\mathrm{G}_{i}$ regulation of secretory vesicle swelling examined by atomic force microscopy. Proc Natl Acad Sci USA 94:13317-13322.

Karnovsky MJ (1965) A formaldehyde-glutaraldehyde fixative of high osmolality for use in electron microscopy. J Cell Biol 27:137A-138A.

Kopell WN, Westhead EW (1982) Osmotic pressures of solutions of ATP and catecholamines relating to storage in chromaffin granules. J Biol Chem 257:5707-5710.

Kozminski KD, Gutman DA, Davila V, Sulzer D, Ewing AG (1998) Voltammetric and pharmacological characterization of dopamine release from single exocytotic events at rat pheochromocytoma (PC12) cells. Anal Chem 70:3123-3130.

Kwok R, Evans E (1981) Thermoelasticity of large lecithin bilayer vesicles. Biophys J 35:637-652.

Liu Y, Schweitzer ES, Nirenberg MJ, Pickel VM, Evans CJ, Edwards RH (1994) Preferential localization of a vesicular monoamine transporter to dense core vesicles in PC12 cells. J Cell Biol 127:1419-1433.

Madeddu L, Saito I, Hsiao TH, Meldolesi J (1985) Leptinotoxin- $h$ action in synaptosomes and neurosecretory cells: stimulation of neurotransmitter release. J Neurochem 45:1719-1730.

O'dowd BF, Seeman P, George SR (1994) In: Handbook of receptors and channels (Peroutka SJ, ed). Boca Raton, FL: CRC.

Ornberg RL, Duong LT, Pollard HB (1986) Intragranular vesicles: new organelles in the secretory granules of adrenal chromaffin cells. Cell Tissue Res 245:547-553. 
Ornberg RL, Furuya S, Goping G, Kuijpers GA (1995) Granule swelling in stimulated bovine adrenal chromaffin cells: regulation by internal granule pH Cell Tissue Res 279:85-92.

Pickel VM, Beckley SC, Joh TH, Reis DJ (1981) Ultrastructural immunocytochemical localization of tyrosine hydroxylase in the neostriatum. Brain Res 225:373-385.

Piehl K, Travis E, Borges R, Wightman RM (1996) Exocytotic release from individual granules exhibits similar properties at mast and chromaffin cells. Biophys J 71:1633-1640.

Pothos E, Desmond M, Sulzer D (1996) L-3,4-Dihydroxyphenylalanine increases the quantal size of exocytotic dopamine release in vitro. J Neurochem 66:629-636.

Pothos EN, Davila V, Sulzer D (1998a) Presynaptic recording of quanta from midbrain dopamine neurons and modulation of the quantal size. J Neurosci 18:4106-4118.

Pothos EN, Przedborski S, Davila V, Schmitz Y, Sulzer D (1998b) D D $_{2}$-like dopamine autoreceptor activation reduces quantal size in PC12 cells. J Neurosci 18:5575-5585.

Pozzan T, Gatti G, Dozio N, Vicentini LM, Meldolesi J (1984) $\mathrm{Ca}^{2+}$. dependent and -independent release of neurotransmitters from PC12 cells: a role for protein kinase C activation? J Cell Biol 99:628-638.

Schafer T, Karli UO, Gratwohl EK, Schweizer FE, Burger MM (1987) Digitonin-permeabilized cells are exocytosis competent. J Neurochem 49:1697-1707.

Scherman D, Henry JP (1984) Reserpine binding to bovine chromaffin granule membranes. Characterization and comparison with dihydrotetrabenazine binding. Mol Pharmacol 25:113-122.

Schroeder TJ, Jankowski JA, Kawagoe KT, Wightman RM, Lefrou C, Amatore C (1992) Analysis of diffusional broadening of vesicular packets of catecholamines released from biological cells during exocytosis. Anal Chem 64:3077-3083.

Schroeder TJ, Borges R, Finnegan JM, Pihel K, Amatore C, Wightman RM (1996) Temporally resolved, independent stages of individual exocytotic secretion events. Biophys J 70:1061-1068.

Schubert D, Klier FG (1977) Storage and release of acetylcholine by a clonal cell line. Proc Natl Acad Sci USA 74:5184-5188.

Schubert D, LaCorbiere M, Klier FG, Steinbach JH (1980) The modulation of neurotransmitter synthesis by steroid hormones and insulin. Brain Res 190:67-79.

Song H, Ming G, Fon E, Bellocchio E, Edwards RH, Poo M (1997) Expression of a putative vesicular acetylcholine transporter facilitates quantal transmitter packaging. Neuron 18:815-826.

Sudhof TC (1982) Core structure, internal osmotic pressure and irreversible structural changes of chromaffin granules during osmometer behavior. Biochim Biophys Acta 684:27-39.
Thureson-Klein A, Klein RL, Chen Yen SH (1975) Morphological effects of osmolarity on purified noradrenergic vesicles. J Neurocytol 4:609-627.

Tischler AS, Greene LA (1978) Morphologic and cytochemical properties of a clonal line of rat adrenal pheochromocytoma cells which respond to nerve growth factor. Lab Invest 39:77-89.

Travis ER, Wang YM, Michael DJ, Caron MG, Wightman RM (2000) Differential quantal release of histamine and 5-hydroxytryptamine from mast cells of vesicular monoamine transporter 2 knock-out mice. Proc Natl Acad Sci USA 97:162-167.

Van der Kloot W (1987) Pretreatment with hypertonic solutions increases quantal size at the frog neuromuscular junction. J Neurophysiol 57:1536-1554.

VanderMeulen J, Grinstein S (1982) $\mathrm{Ca}^{2+}$-induced lysis of platelet secretory granules. J Biol Chem 257:5190-5195.

Varoqui H, Erickson JD (1997) Vesicular neurotransmitter transporters. Potential sites for the regulation of synaptic function. Mol Neurobiol 15:165-191.

Venable JH, Coggeshall R (1965) A simplified lead citrate stain for use in electron microscopy. J Cell Biol 25:407-408.

Videen JS, Mezger MS, Chang YM, O'Connor DT (1992) Calcium and catecholamine interactions with adrenal chromogranins. Comparison of driving forces in binding and aggregation. J Biol Chem 267:3066-3073.

Wagner JA (1985) Structure of catecholamine secretory vesicles from PC12 cells. J Neurochem 45:1244-1253.

Watanabe O, Torda M, Meldolesi J (1983) The effect of $\alpha$-latrotoxin on the neurosecretory PC12 cell line: electron microscopy and cytotoxicity studies. Neuroscience 10:1011-1024.

Wightman RM, Jankowski JA, Kennedy RT, Kawagoe KT, Schroeder TJ, Leszczyszyn DJ, Near JA, Diliberto Jr EJ, Viveros OH (1991) Temporally resolved catecholamine spikes correspond to single vesicle release from individual chromaffin cells. Proc Natl Acad Sci USA 88:10754-10758.

Wightman RM, Schroeder TJ, Finnegan JF, Ciolkowski EL, Pihel K (1995) Time-course of release of catecholamine from individual vesicles during exocytosis at adrenal medullary cells. Biophys J 68:383-390.

Winkler H, Westhead E (1980) The molecular organization of adrenal chromaffin granules. Neuroscience 5:1803-1823.

Yoo SH, Albanesi JP (1991) High-capacity, low-affinity $\mathrm{Ca}^{2+}$ binding of chromogranin A. Relationship between the $\mathrm{pH}$-induced conformational change and $\mathrm{Ca}^{2+}$ binding property. J Biol Chem 266:7740-7745.

Zimmerberg J, Curran M, Cohen FS, Brodwick M (1987) Simultaneous electrical and optical measurements show that membrane fusion precedes secretory granule swelling during exocytosis of beige mouse mast cells. Proc Natl Acad Sci USA 84:1585-1589. 